\title{
The Spherical Atom Model of Helium Based on the Theorem of Niels Bohr
}

\author{
Thomas Allmendinger \\ Independent Scholar, Zurich, Switzerland \\ Email: inventor@sunrise.ch
}

How to cite this paper: Allmendinger, T. (2019) The Spherical Atom Model of Helium Based on the Theorem of Niels Bohr. Journal of Applied Mathematics and Physics, 7, 172-180. https://doi.org/10.4236/jamp.2019.71015

Received: December 7, 2018

Accepted: January 20, 2019

Published: January 23, 2019

Copyright $\odot 2019$ by author(s) and Scientific Research Publishing Inc. This work is licensed under the Creative Commons Attribution International License (CC BY 4.0).

http://creativecommons.org/licenses/by/4.0/

(c) (i) Open Access

\begin{abstract}
Proceeding from the double-cone model of Helium, based on Bohr's theorem and recently published in [13], a spherical modification could be made by introducing a second electron rotation which exhibits a rotation axis perpendicular to the first one. Thereby, each rotation is induced by the spin of one electron. Thus the trajectory of each electron represents the superposition of two separate orbits, while each electron is always positioned opposite to the other one. Both electron velocities are equal and constant, due to their mutual coupling. The $3 \mathrm{D}$ electron orbits could be $2 \mathrm{D}$-graphed by separately projecting them on the $\mathrm{x} / \mathrm{z}$-plane of a Cartesian coordinate system, and by plotting the evaluated $x-, y-$ and $z$-values versus the rotation angle. Due to the decreased electron velocity, the resulting radius is twice the size of the one in the double-cone model. Even if distinct evidence is not feasible, e.g. by means of X-ray crystallographic data, this modified model appears to be the more plausible one, due to its higher cloud coverage, and since it comes closer to Kimball's charge cloud model.
\end{abstract}

\section{Keywords}

Modified Bohr Model, Electron-Trajectories, Electron-Spin, 3D-Atom-Model, Confutation of Heisenberg's Uncertainty-Principle

\section{Introduction}

The hydrogen atom model of Niels Bohr, published in 1913 [1], and based on Max Planck's quantum theory of light, published in 1900 [2] and supplemented by Albert Einstein's photoelectric effect in 1905 [3], represented a cardinal milestone in understanding the structure of matter. This atom model could explain the mathematical regularities of the absorption- and emission-lines in the discrete UV-spectrum of hydrogen, first discovered by Balmer already in 1885 [4], 
and later revealed by Rydberg. The explanation of these regularities is given by assuming definite electron orbits around the nucleus, characterized by integer multiples of its angular momentum $h / 2 \pi$ in the ground state. However, the real cause for this multiplicity could not be found. Moreover, the existence of such a ground state (Bohr called it "permanent state") could not be explained. It should be noticed that Bohr's electron orbits correspond to energetically excited electronic states, and not to electron shells which are commonly assumed for atoms with higher atomic numbers, explaining the "aufbau-principle" of the periodic system, and solely concerning the ground states of the atoms.

Ten years later, the hypothesis of Louis de Broglie allowed taking a step forward, assuming the wavy nature of electron trajectories, and implying standing electron waves in their excited states. However, such well-defined trajectories could not be vividly evaluated at that time. As a consequence, Heisenberg postulated the so-called "uncertainty principle", implying for each electron probabilities of presence, instead of well-describable trajectories. This assumption was adopted by the leading physicists, especially by Born, Schrödinger and Dirac. 90 years later, it still represents the "official" quantum mechanical doctrine, even if it contradicts the fundamental scientific principle of causality, ignoring the existence of an angular momentum in the ground state, disregarding the fact that standing waves represent the epitome of accuracy, and hazarding the lacking vividness and unintelligibility of that model. After all, the charge cloud model of Kimball, proposed in 1940 [5], delivered an improved visualisation, qualitatively explaining the atomic structure of the elements as well as the valences in molecular bonds. It is well usable in chemistry, but it does not exactly render the original quantum mechanical approach.

Based on the multiplicities of spectral lines found in the presence of magnetic fields, Uhlenbeck and Goudsmith postulated in 1925 the electron spin [6] [7]. This phenomenon was implemented afterwards in the already existing theory, introducing the Pauli-principle. However, it is of extreme importance for the whole quantum mechanical reception since it explains the existence of the basic angular momentum in the Bohr model due to a-empirically detectable-coupling with the spin of the electron. So it must constitute the theory and cannot be added afterwards to a basically insufficient theory. When the spin and its coupling with the electron orbit are regarded from the outset, Heisenberg's uncertainty principle becomes unnecessary and can thus be abandoned. Nevertheless, unintelligibility remains since the existence of the spin cannot be classically explained. It is unalterable, like the elemental charge.

Induced by these contradictions, the author searched and found a vivid solution for the excited electron states implying the De Broglie phenomenon and starting from Bohr's original approach of the $\mathrm{H}$-atom-model [8]. Thereby, it was needed to assume three-dimensional wavy electron trajectories in the excited states, winding up on a surface similar to one of a hyperboloid (Figure 1), whereas at the ground state the electron trajectory is planar. Thereby, the most 
delicate sticking point was given by the fact that the partial horizontal angular momentum (assigned to the rotation axis in Figure 1) remains constant even in the exited states, while the total angular momentum obeys Bohr's theorem.

Initially, the existence of the (stable) planar ground state could not be explained. But after having found its real cause-namely the electron spin-the way was clear for modelling molecules and atoms in the ground state. In particular, it was possible to develop a vivid model for the $\mathrm{H}_{2}$-molecule, exhibiting planar electron orbits [9]. Analogously to the conventional method of Heitler and London [10] [11], the bond length was computed by searching the total energy minimum (Figure 2). Since the bond length can be directly determined by

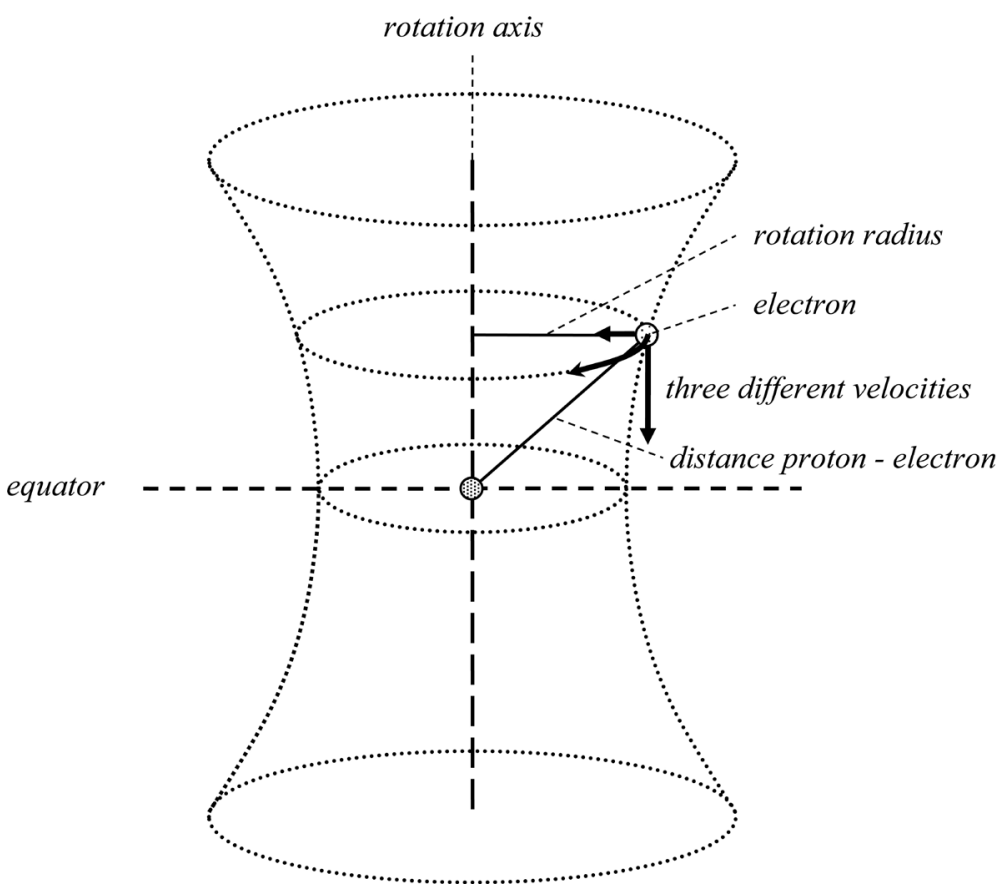

Figure 1. Intermediate position of the electron at the modified model according to [8].

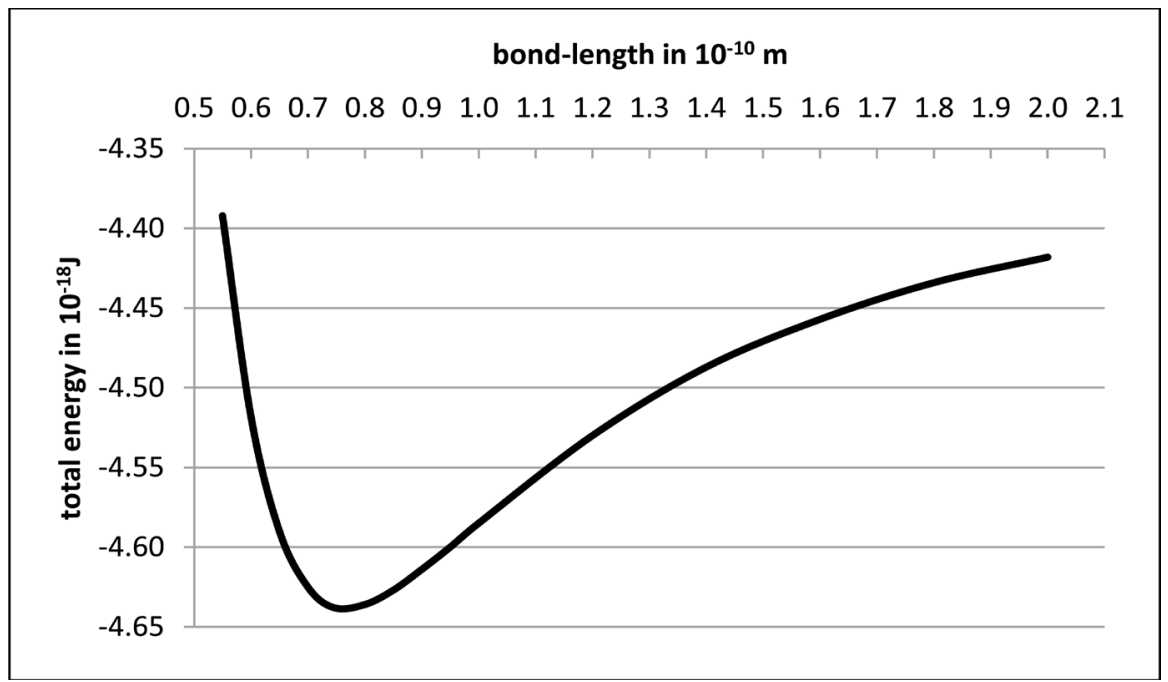

Figure 2. Total energy as a function of the bond-length at the $\mathrm{H}_{2}$-molecule. 
$\mathrm{X}$-ray measurements, verification was possible by empirical evidence, delivering an accurate result (Figure 3). In contrast to this, the results of Heitler and London-as well as those of others [12] - delivered variable and less accurate results. Thus a second proof for the existence of well-defined electron-trajectories could be delivered-namely by explaining the ground-state of the $\mathrm{H}_{2}$-molecule-, in contrast to the conventional theory.

Extending this approach, atom models for the noble gases Helium and Neon were developed, keeping in mind Kimball's perception [13]. But aside from the fact, that precise empirical evidence could not be delivered because of the absence of directed covalent bonds, for Neon an exact model computation was not feasible in view of the complexity of the problem. Beyond that, meanwhile doubts have risen about the plausibility of these models, even about the one of Helium, partially-but not entirely-questioning this original approach, and leading to an improved model approach for Helium which is subject of this article.

The essential idea of the original approach consisted in the assumption of an eccentric structure of the electron orbits leading to a three-dimensional double-cone (Figure 5), instead of a concentric planar ring (Figure 4). The computation of

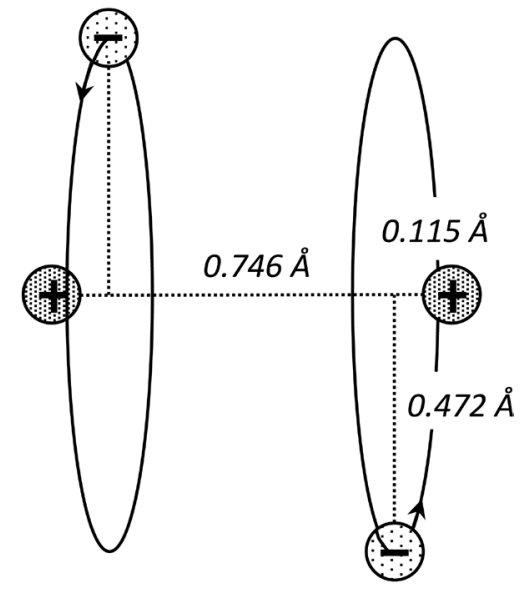

Figure 3. Model of the $\mathrm{H}_{2}$-molecule (true to scale).

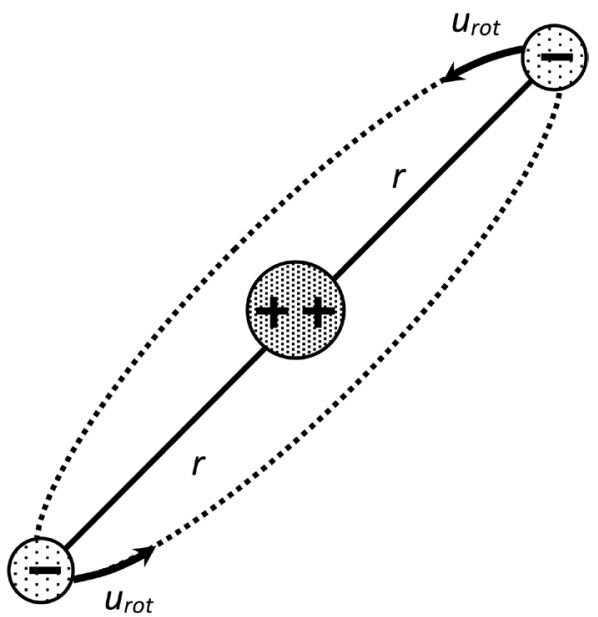

Figure 4. Concentric atom model of Helium. 


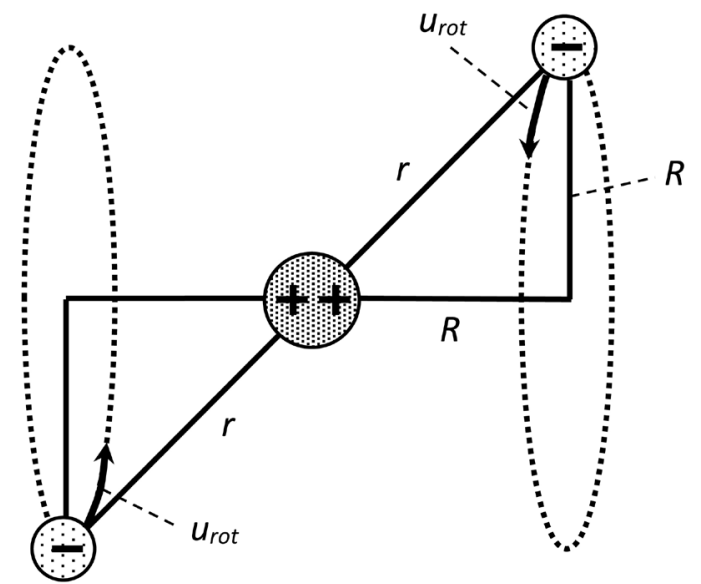

Figure 5. Eccentric double-cone atom model of Helium.

the radius $r$ yielded the value

$$
r=\frac{8 h^{2} \cdot \varepsilon_{0}}{7 \pi \cdot m_{e} \cdot e^{2}}=0.60477 \times 10^{-10} \mathrm{~m}
$$

(Ad [13]: In Equation (4), the quotient 8 should be substituted by 4, but the result is correct)

However, this electron array does not resemble a sphere which would be desirable according to Kimball's perception, and which would explain the atomic cores of the higher elements as well as the close-packing of ions in crystal lattices. Moreover, it appears odd that the spins of the two electrons refer in the same manor to the nucleus, inducing the same angular momentum. As a consequence, this model has been modified in the following way.

\section{The Spherical Modification of the Double-Cone Model}

The double-cone model of Helium can be morphed into a spherical one by introducing a second electron rotation which exhibits a rotation axis perpendicular to the first one. Thereby, each rotation is induced by the spin of one electron. Thus the trajectory of each electron represents the superposition of two separate orbits, while each electron is always positioned opposite to the other one. Both electron velocities are equal and constant, due to mutual coupling (Figure 6).

In order to $2 D$-describe the electron trajectories in a Cartesian coordinate system with the rotation angle $\varphi$ as a variable, their $x^{-}, y$ - and $z$-projections on the $x / z$-plane may be used as parameters. Applying trigonometric relations, for one electron the analysis yields

$$
x=R\left((\sin \varphi)^{2}-\cos \varphi\right), y=R \cdot \sin \varphi(1+\cos \varphi), \quad z=-R \cdot \cos \varphi
$$

For the other electron, the formulas are identical equal except the sign. The respective diagrams for the two electrons are shown in the Figure 7 and Figure 8.

The numerical computation of $R$ and $r$ can be made analogously to the one for the double-cone case, described in [13]. However, in the expression for the centrifugal force, which is relevant for the force equilibrium, $u_{\text {rot }}^{2}$ has to be substituted by 


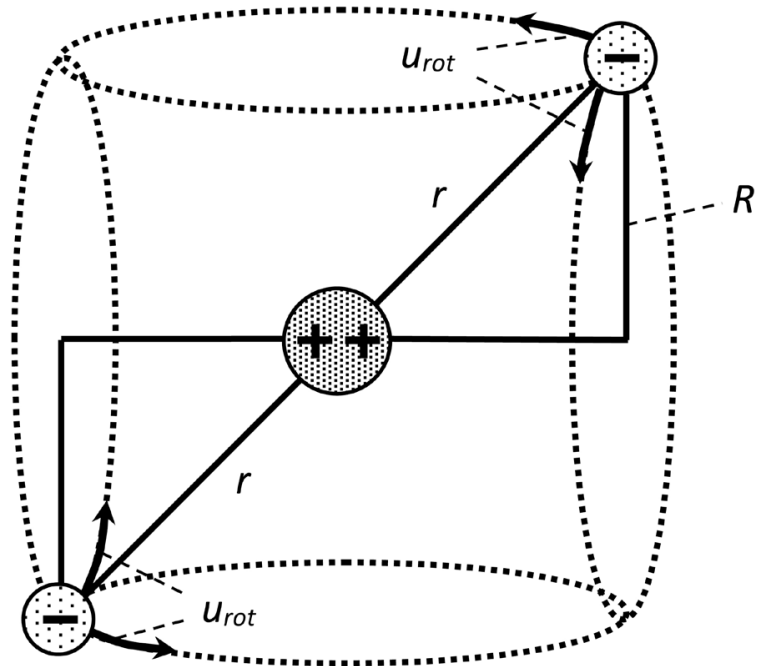

Figure 6. Spherical model of Helium.

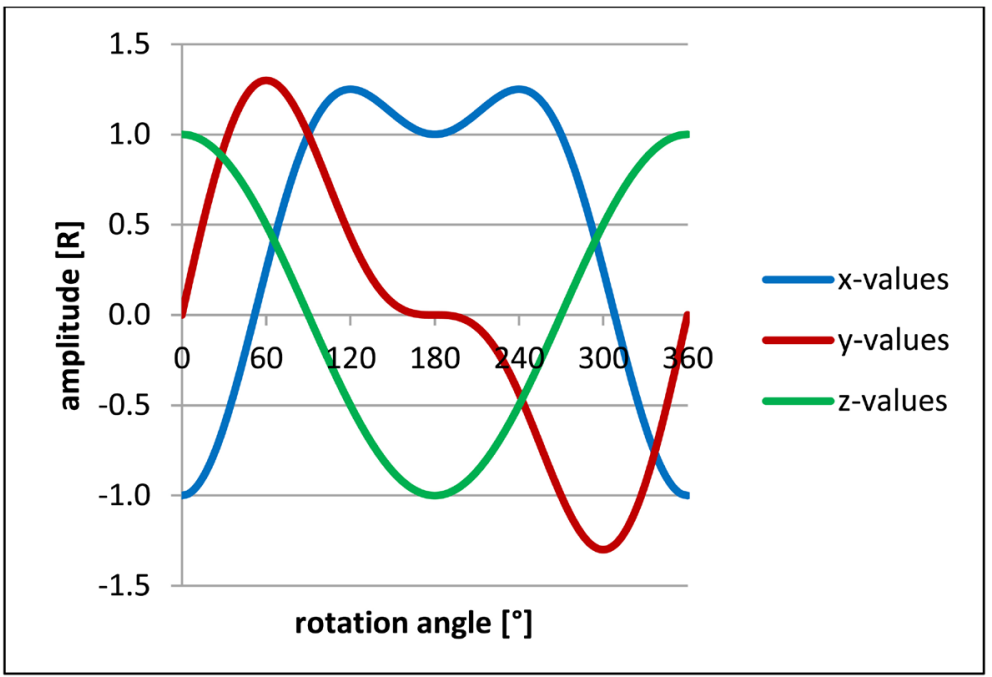

Figure 7.2D-diagram of one electron.

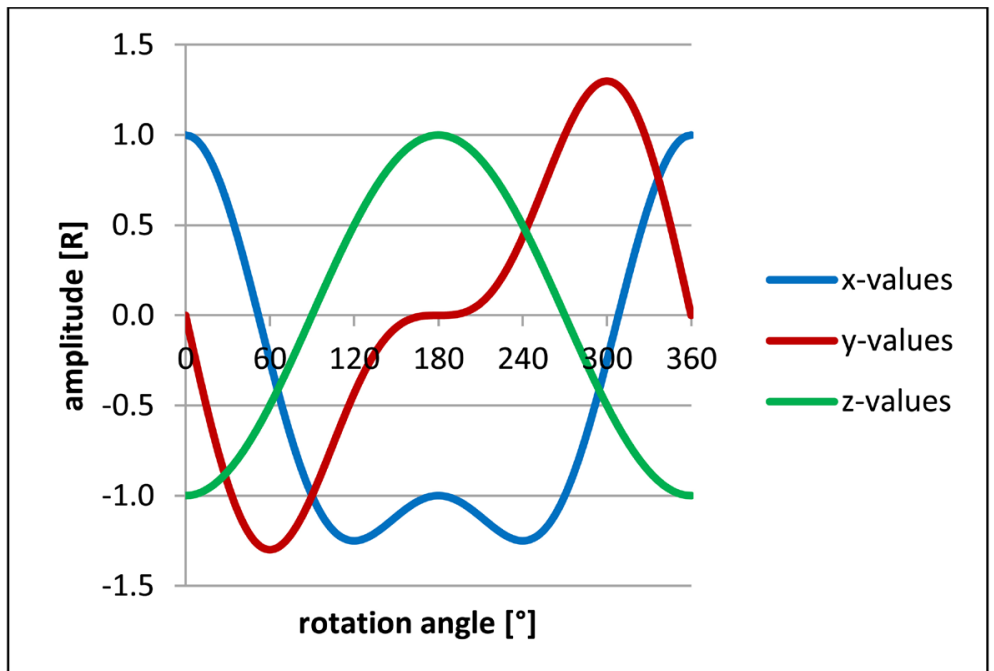

Figure 8. 2D-diagram of the other electron. 
$u_{t o t}^{2}=2 u_{r o t}^{2}$.

Coulomb-attraction between the nucleus and an electron: $\frac{2 K}{r^{2}}$

whereby $K=\frac{e^{2}}{4 \pi \varepsilon_{0}}$

Coulomb-repulsion between the two electrons: $\frac{K}{4 r^{2}}$

Centrifugal force:

$\frac{2 m_{e} \cdot u_{r o t}^{2}}{r}$

Force equilibrium:

$$
\frac{2 K}{r^{2}}=\frac{K}{4 r^{2}}+\frac{2 m_{e} \cdot u_{r o t}^{2}}{r}
$$

Quantum condition for the angular momentum of an electron:

$m_{e} \cdot u_{r o t} \cdot R=\frac{h}{2 \pi}$

Since $R=r \sqrt{2}$, the calculation yields $r=\frac{16 h^{2} \cdot \varepsilon_{0}}{7 \pi \cdot m_{e} \cdot e^{2}}=1.21 \times 10^{-10} \mathrm{~m}$, and $R=0.855 \times 10^{-10} \mathrm{~m}$.

Due to the increased electron velocity, the result is twice as high as the one in the double-cone model.

\section{Summary and Conclusions}

Proceeding from the double-cone model of Helium, based on Bohr's theorem and recently published in [13], a spherical modification could be made by introducing a second electron rotation which exhibits a rotation axis perpendicular to the first one. Thereby, each rotation is induced by the spin of one electron. Thus the trajectory of each electron represents the superposition of two separate orbits, while each electron is always positioned opposite to the other one. Both electron velocities are equal and constant, due to their mutual coupling. The $3 \mathrm{D}$ electron orbits could be $2 \mathrm{D}$-graphed by separately projecting them on the $\mathrm{x} / \mathrm{z}$-plane of a Cartesian coordinate system, and by plotting the evaluated $\mathrm{x}-, \mathrm{y}-$ and $\mathrm{z}$-values versus the rotation angle.

Due to the increased electron velocity, the resulting radius is twice the size of the one in the double-cone model. Even if distinct evidence is not feasible, e.g. by means of X-ray crystallographic data, this modified model appears to be the more plausible one, due to its higher cloud coverage, and since it comes closer to Kimball's charge cloud model. Contrary to the conventional quantum mechanical approach assuming a diffuse 1s-orbital with temporally variable electron radii, this spherical Helium atom model exhibits precise electron trajectories. Thus it delivers a further proof that Bohr's concept, combined with the spin-theorem, and implicating the spin-orbit coupling, can be employed on any case, even if for more complicated atoms or molecules the calculation seems quite intricate. Moreover, it represents a vivid explanation of the Pauli Principle.

The deformation of this atomic electron shell, which is to be expected in the case of collisions due to thermal motion in the gas state, has not been studied so 
far. At least, the author's recent investigations about the thermal behaviour of gases under the influence of infrared-radiation gave evidence enough that even at noble gases absorption as well as emission of thermal radiation takes place [14], which must be attributed to energetic excitations of the electron shells.

\section{Conflicts of Interest}

The author declares no conflicts of interest regarding the publication of this paper.

\section{References}

[1] Bohr, N. (1913) On the Constitution of Atoms and Molecules. The London, Edinburgh, and Dublin Philosophical Magazine and Journal of Science, 26, 1-25. https://doi.org/10.1080/14786441308634955

[2] Planck, M. (1900) Ueber Irreversible Strahlungsvorgänge. Annals of Physics, 306, 69-116. https://doi.org/10.1002/andp.19003060105

[3] Einstein, A. (1905) Ueber einen die Erzeugung und Verwandlung des Lichts betreffenden heuristischen Gesichtspunkt. Annals of Physics, 322, 132-148. https://doi.org/10.1002/andp.19053220607

[4] Balmer, J.J. (1885) Notiz über die Spectrallinien des Wasserstoffs. Annalen der Physik, 25, 80-87. https://doi.org/10.1002/andp.18852610506

[5] Kimball, G.E. (1940) Directed Valence. Journal of the Physical Chemistry, 8, 188. https://doi.org/10.1063/1.1750628

[6] Uhlenbeck, G.E. and Goudsmith, S. (1925) Ersetzung der Hypothese vom unmechanischen Zwang durch eine Forderung bezüglich des inneren Verhaltens jedes einzelnen Elektrons. Naturwissenschaften, 13, 953.

[7] Uhlenbeck, G.E. and Goudsmith, S. (1926) Spinning Electrons and the Structure of Spectra. Nature, 117, 264-265. https://doi.org/10.1038/117264a0

[8] Allmendinger, T. (2016) A Classical Approach to the De Broglie-Wave Based on Bohr's H-Atom-Model. International Journal of Applied Mathematics and Theoretical Physics, 2, 1-15.

http://www.sciencepublishinggroup.com/journal/paperinfo?journalid=322\&doi=10. 11648/j.ijamtp.20160201.11

[9] Allmendinger, T. (2018) The Elucidation of the Ground State in the H-Atom-Model of Niels Bohr and Its Application on the Bond-Length Computation in the $\mathrm{H}_{2}$ Molecule. International Journal of Molecular and Theoretical Physics, 2, 1-10.

https://symbiosisonlinepublishing.com/molecular-theoretical-physics/molecular-th eoretical-physics09.pdf https://doi.org/10.15226/2576-4934/2/1/00109

[10] Heitler, W. and London, F. (1927) Wechselwirkung neutraler Atome und homöopolare Bindung nach der Quantenmechanik. Zeitschrift für Physik, 44, 455-472. https://doi.org/10.1007/BF01397394

[11] Heitler, W. (1961) Elementare Wellenmechanik. Friedr. Vieweg \& Sohn, Braunschweig.

[12] Haken, H. and Wolf, H.C. (2006) Molekülphysik und Quantenchemie. Springer.

[13] Allmendinger, T. (2018) The Atom Model of Helium and of Neon Based on the Theorem of Niels Bohr. Journal of Applied Mathematics and Physics, 6, 1290-1300. https://www.scirp.org/Journal/PaperInformation.aspx?PaperID=85615 https://doi.org/10.4236/jamp.2018.66108 
[14] Allmendinger, T. (2016) The Thermal Behaviour of Gases under the Influence of Infrared-Radiation. International Journal of Physical Sciences, 11, 183-205. https://academicjournals.org/journal/IJPS/article-full-text-pdf/E00ABBF60017 https://doi.org/10.5897/IJPS2016.4500 